Proceedings

\title{
Biocompatible Temperature Nanosensors Based on Titanium Dioxide ${ }^{+}$
}

\author{
Veronica Zani ${ }^{1}$, Danilo Pedron ${ }^{1}$, Roberto Pilot ${ }^{2}$ and Raffaella Signorini ${ }^{2, *}$ \\ 1 University of Padua; veronica.zani@unipd.it (V.Z.); danilo.pedron@unipd.it (D.P.) \\ 2 University of Padua and Consorzio INSTM; roberto.pilot@unipd.it \\ * Correspondence: Raffaella.signorini@unipd.it; Tel.: +39-049-8275118 \\ + Presented at the 1st International Electronic Conference on Biosensors, 2-17 November 2020; Available \\ online: https://iecb2020.sciforum.net/.
}

Received: date; Accepted: date; Published: date

\begin{abstract}
The measurement of temperature is of fundamental importance in a huge scale of applications, from nanomedicine, where the early detection of tumorous cells is an essential requirement, to microelectronics and microcircuits [1]. Optical sensors with a micro/nano-spatial resolution can be used for temperature determination within a biological frame. Within this contest, Raman spectroscopy [2] is particularly interesting: the inelastic scattering of light, has the advantage of a contactless measurement and exploits the temperature-dependence of intensities in the spectrum by observing the intensity ratio of anti-Stokes and Stokes signals. Titanium Dioxide can be regarded as a potential optical material for the temperature detection in biological samples, thanks to its high biocompatibility, already demonstrated in literature [3], and to its strong Raman scattering signal. The aim of the present work is the realization of biocompatible optical thermometers, with a sub-micrometric spatial resolution, made of Titanium dioxide. Raman measurements have been performed on Anatase powder using 514.5, 568.2 and $647.1 \mathrm{~nm}$ excitation lines of the $\mathrm{CW} \mathrm{Ar} / \mathrm{Kr}$ ion laser. The laser beam is focalized through a microscope on the sample, kept at defined temperature using a temperature controller. The Stokes and anti-Stokes scattered light is analysed through a triple monochromator and detected by a liquid nitrogen cooled CCD camera. Raw data are analysed with Matlab and Raman spectrum parameters-like area, intensity, frequency position and width of the peak-are calculated using a pseudo-Voight fitting curve. Preliminary results showed that good reliable temperatures can be obtained.
\end{abstract}

Keywords: Raman spectroscopy; temperature; titanium dioxide

\section{Introduction}

The field of temperature sensors application is wide and varied, ranging from the measurement of temperature in biological samples and tissues to the detection of hot spots in microcircuits [1]. In particular biological applications [4-7] require high temperature resolution, high spatial resolution and proven biocompatibility of the material chosen as sensor.

Among all techniques available nowadays for the temperature measurement with the requirement of nano and micrometric spatial resolution, Raman spectroscopy [8-10] has the great advantage of a contactless measurement, moreover it does not require a specific preparation of the samples. With respect to other optical thermometric techniques, as IR thermography, it's characterized by a greater spatial resolution, on the order of the diffraction limit of the probe laser [11].

The Raman effect is the inelastic scattering of light and well defined characteristics of the Raman spectrum, like intensity, position in frequency and width of peak signals, are related to temperature. 
It follows that temperature can be measured from Raman spectra by determining the degree of the shift position of a defined peak at different temperatures, or by evaluating the broadening of its linewidth or by measuring the peak intensity ratio of the anti-Stokes signal to the Stokes signal. In the present work the anti-Stokes/Stokes intensity ratio is used to estimate the local temperature of the material [12].

A good Raman thermometer material should possess these properties: (a) a large Raman scattering cross-section (to reach high signal-to-noise ratios), (b) high intensity Raman peaks at low Raman shifts, the upper limit depends on the working temperature, in general near room temperature it is about $600 \mathrm{~cm}^{-1}$ [10]; indeed the lower the Raman shift the more sensitive is the peak intensity to temperature, (c) well-defined and distinguishable Raman peaks, (d) low absorbance at the excitation wavelength, to avoid heating mechanisms.

Studies of Raman temperature measurement can be found in literature for Silicon [11,13], Gallium Arsenide [14], Gallium Nitride [15] and graphene [16]. Also Titanium dioxide $\left(\mathrm{TiO}_{2}\right)$ has been tested in few works as a Raman temperature thermometer for Titanium dioxide microparticles $[17,18]$ and for thin films of Titania used in solar cells [19].

Titanium dioxide general features of chemical stability and nontoxicity, make it a very interesting compound for various different applications, including photocatalysis $[8,20]$, optical coatings, optoelectronic devices [21] and biomedicine [3]. It is a wide band gap insulator (3.0 eV [22]) and exists in nature in three different crystal structures: anatase, rutile and brookite. In particular the anatase phase is exploited in photocatalysis, photochemical solar cells, optoelectronic devices, and chemical sensors $[23,24]$. Titanium dioxide seems to suite perfectly all the requirements for a good thermometer material and has been chosen as Raman active thermometric material in our research.

\section{Materials and Methods}

Raman measurements have been performed using a Micro-Raman setup in a back-scattering geometry. The system is equipped with a CW Ti:Sapphire Laser tunable in the range 675-1000 nm (Spectra Physics, 3900S) pumped by a CW Optically Pumped Semiconductor Laser (Coherent, Verdi G7) and an $\mathrm{Ar}^{+} / \mathrm{Kr}^{+}$gas laser (Coherent, Innova 70) providing the lines at 488, 514.5, 530.8, 568 and $647.1 \mathrm{~nm}$. The laser beam is coupled to a microscope (Olympus BX 40) and focused on the sample by a $100 \times, 50 \times$ or $20 \times$ objectives (Olympus SLMPL). The Raman scattering is collected into the slit of a three stages subtractive spectrograph (Jobin Yvon S3000) by means of a set of achromatic lenses. The spectrograph is made up by a double monochromator (Jobin Yvon, DHR 320), working as a tunable filter rejecting elastic scattering, and a spectrograph (Jobin Yvon, HR 640). The Raman signal is detected by a liquid nitrogen cooled CCD (Jobin Yvon, Symphony $1024 \times 256$ pixels front illuminated).

A temperature controlled stage (Linkam, THMS600/720) is used to change the temperature of the sample, by means of a liquid nitrogen reservoir and heating resistances. The sample, a commercial Anatase powder (Sigma Aldrich), is inserted in the temperature controller stage, and uniformly heated/cooled to reach the desired temperature, with a rate of $5^{\circ} \mathrm{K} / \mathrm{min}$ and a thermalization time of at least $30 \mathrm{~min}$. Before starting the experiment, a procedure of purging air from the stage chamber with nitrogen is done. Once the thermalization process is done, consecutive Stokes and anti-Stokes measurements are conducted to calculate the local temperature of the sample. Measurements are repeated to obtain a consistent set of data. The frequency incident on the sample is properly chosen in order to avoid sample heating, that is by using photons less energetics than the band gap of Titanium dioxide [22]. Measurements of the anti-Stokes/Stokes ratio at different laser powers confirm that the signal is independent by power over the range explored, i.e., $0.5-20 \mathrm{~mW}$. Usually for temperature measurements a power of $3-4 \mathrm{~mW}$ is used. 


\section{Results and discussion}

\subsection{Characterization of $\mathrm{TiO}_{2}$ through Raman Spectroscopy}

The Raman spectrum of Anatase powder, recorded at room temperature, using a laser at 647.1 $\mathrm{nm}$, with an input power of $1.52 \mathrm{~mW}$ is reported in Figure 1. The spectrum clearly shows an intense peak centred at $143 \mathrm{~cm}^{-1}$ and peaks with a lower intensity that are outlined in the insets.
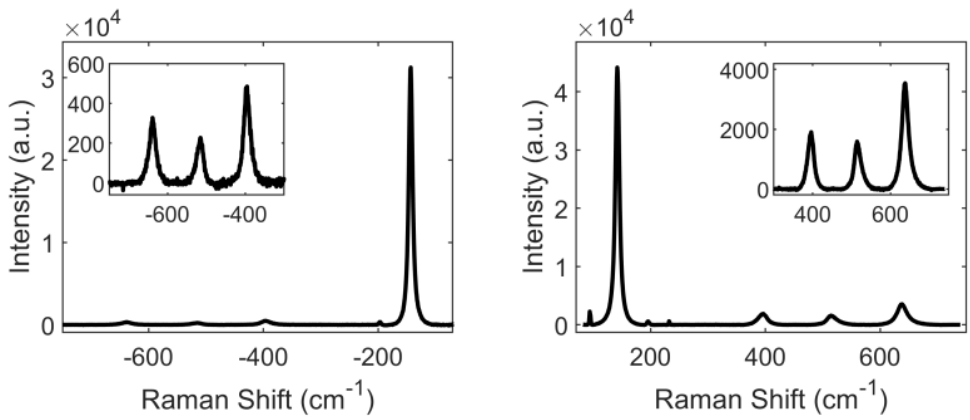

Figure 1. Stokes (right) and anti-Stokes (left) Raman spectrum of Titanium dioxide anatase at 647.1 nm.

Anatase is characterized by 15 optical modes at the $\Gamma$ point of the Brillouin zone, described with the following irreducible representation of the normal vibrational modes [21,25]:

$$
1 A_{1 g}+1 A_{2 u}+2 B_{1 g}+1 B_{2 u}+3 E_{g}+2 E_{u}
$$

Among all these, six modes, $A_{1 g}, 2 B_{1 g}$, and $3 E_{g}$ are the Raman-active ones (as reported in Figure 1 and in Table 1); the Raman spectra show only five peaks since the $B_{1 g}$ and $A_{1 g}$ modes at 512 and $518 \mathrm{~cm}^{-1}$ are not distinguishable at the experimental conditions. The experimental frequencies, reported in Table 1, have been corrected by using the cyclohexane frequencies as calibration, and are comparable to data reported in literature [21].

Table 1. Experimental Titanium dioxide anatase Raman-active modes compared to those reported in literature [21].

\begin{tabular}{ccc}
\hline Symmetry & Experimental $\mathbf{( \mathbf { c m } ^ { - 1 } )}$ & Literature [21] $\left.\mathbf{( c m}^{-\mathbf{1}}\right)$ \\
\hline$E_{g}$ & 143 & 143 \\
$E_{g}$ & 197 & 198 \\
$B_{1 g}$ & 397 & 395 \\
$B_{1 g}$ & 515 & 512 \\
$A_{1 g}$ & & 518 \\
$E_{g}$ & 640 & 639 \\
\hline
\end{tabular}

By observing Stokes and anti-Stokes data, it turned out that the best peak for temperature monitoring is the $E_{g}$ mode at $143 \mathrm{~cm}^{-1}$. It is well defined, very intense even at low laser powers, and highly sensitive to temperature, thanks to its low Raman shift.

\section{Temperature Determination}

Raw Raman data are analysed with Matlab, using a Voigt fitting, to obtain the Raman spectrum parameters, like area, intensity, frequency position and width of the peak. In particular, the antiStokes area of the $E_{g}$ mode is divided by that of the Stokes signal to obtain the experimental antiStokes/Stokes ratio, $\rho$, reported in Table 2 , on the second column. 
Table 2. Anti-Stokes/Stokes ratios and row temperatures determined from Raman experimental data, measured at $298 \mathrm{~K}$, at different the excitation wavelengths (II and III columns). Values of the calibration constant at the used excitation wavelengths (IV column). Sample temperature obtained from experimental anti-Stokes/Stokes ratios, using the calibration constant (V column).

\begin{tabular}{ccccc}
\hline $\begin{array}{c}\text { Excitation Wavelength } \\
(\mathbf{n m})\end{array}$ & $\begin{array}{c}\text { Anti-Stokes/Stokes } \\
\text { Ratio }\end{array}$ & $\begin{array}{c}\text { Row } \\
\text { Temperature } \\
(\mathbf{K})\end{array}$ & $\begin{array}{c}\text { Calibration } \\
\text { Constant }\end{array}$ & $\begin{array}{c}\text { Sample } \\
\text { Temperature } \\
\text { (K) }\end{array}$ \\
\hline 514.5 & 0.5231 & 292 & $0.979 \pm 0.006$ & 301 \\
568.2 & 0.5120 & 281 & $0.964 \pm 0.007$ & 296 \\
647.1 & 0.6569 & 425 & $1.22 \pm 0.01$ & 298 \\
\hline
\end{tabular}

The anti-Stokes/Stokes ratio allows to determine the local temperature $T$ of the sample, through the relation:

$$
\rho=\frac{I_{a S}}{I_{S}}=C \cdot \frac{\left(v_{m}-v_{0}\right)^{3}}{\left(v_{m}+v_{0}\right)^{3}} \exp \left(-\frac{h v_{m}}{k_{B} T}\right)
$$

where $v_{m}$ is the frequency of the vibrational mode $m$ considered, $v_{0}$ the laser frequency, $h$ the Planck's constant, $k_{B}$ the Boltzmann constant, and $C$ is the calibration constant. This calibration constant is related to the experimental set-up, to parameters like the polarization of the incident laser and the CCD and grating efficiency. It has been determined at each excitation wavelength by performing measurements at temperature variable in the range $283-323 \mathrm{~K}$. The Raman spectra of the lower-frequency $E_{g}$ mode recorded at three temperatures, 283, 298 and $318 \mathrm{~K}$, using an excitation source $\lambda_{\text {exc }}=647.1 \mathrm{~nm}$, are reported in Figure 2a and anti-Stokes/Stokes ratios used for the calibration are plotted in Figure 2b. Equation (1) was fitted to the experimental values, leaving $C$ as a free parameter, and the resulting curve is the solid line shown in Figure $2 \mathrm{~b}$.

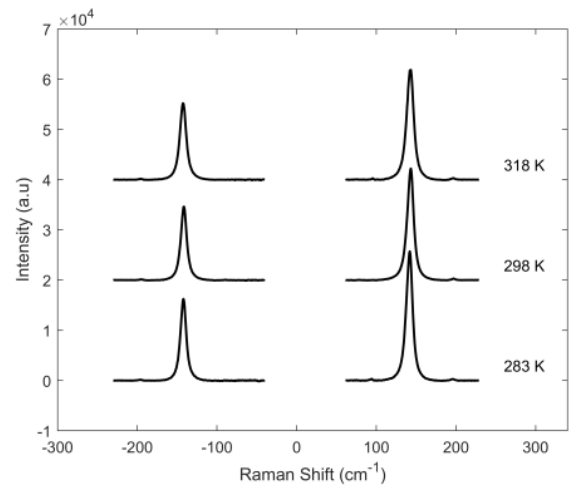

(a)

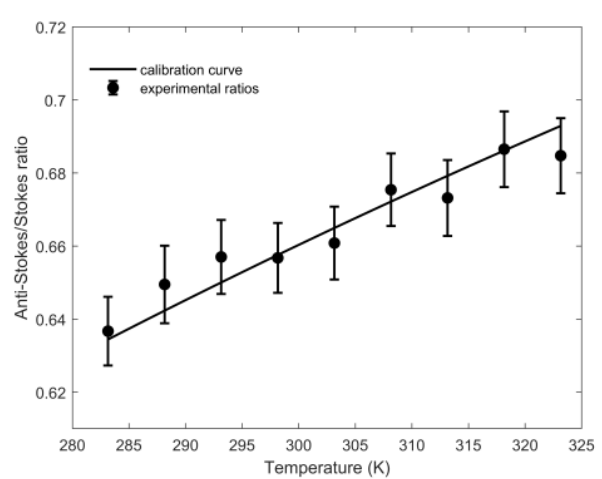

(b)

Figure 2. (a) Raman spectra of the lower-frequency $E_{g}$ mode recorded at 283, 298 and $318 \mathrm{~K}$, using excitation source of $647.1 \mathrm{~nm}$, (b) anti-Stokes/Stokes experimental ratios (open circles) as function of the temperature imposed by the thermostat and calibration curve (solid line).

The same calibration procedure was applied also at 514.5 and $568.2 \mathrm{~nm}$ excitation wavelengths and the values of $C$, with relative uncertainties, are reported in the fourth column of Table 2 . The calibration constant of $0.979,0.964$ and 1.22, respectively at 514.5, 568.2 and $647.1 \mathrm{~nm}$, has been determined with a precision of approximately $0.6-0.8 \%$ (less than $1 \%$ ). Considering that the intensity of the peak can be determined by means of a Voigt fit with a precision of $1 \%$, the total uncertainty on the temperature measurement can be as good as $5 \mathrm{~K}$.

The goodness of the calibration was evaluated on the base of room temperature measurements (at $298 \mathrm{~K}$ ) on the same sample of Titanium dioxide powder, realized in different days. The mean values obtained from the Raman spectra acquired at room temperature using the excitation wavelengths of 514.5, 568.2 and $647.1 \mathrm{~nm}$ are reported in Table 2. The second and the third columns 
report the experimental anti-Stokes/Stokes ratios and the corresponding raw temperatures. These results display a marked difference between temperature measurements at different wave lengths, outlining the importance of the calibration coefficient which considers the instrumental response function, i.e., the efficiency of detection of photons, at diverse wavelengths. The last column reports finally the measured temperature of the sample: the temperature values obtained at different wavelengths are very close to the attended one, $298 \mathrm{~K}$.

\section{Conclusions}

The determination of local temperature at nanoscale is a key point to reach the control of physical, chemical or biological processes, since temperature is an important parameter influencing processes themselves. A great experimental effort is worth to be devoted in the nanothermometry field, from the realization of new materials, to the implementation of detection techniques and test of prototypes of real devices.

In this work Titanium dioxide is proposed as a Raman thermometer for biological applications, as it seems to be a good thermometer material, thanks to its optical and biological properties. $\mathrm{TiO}_{2}$, as Anatase, has been tested in a wide optical range, at wavelengths of 514.5, 568.2 and $647.1 \mathrm{~nm}$, where it has been demonstrated its efficiency in the temperature detection, which can be obtained following the identification of the instrumental calibration coefficients.

Actually, these preliminary results achieved are promising, and represent a starting point for future experiments, regarding the investigation in a wider wavelength range, close to the biological window, or the realization of more complex titanium dioxide based systems.

\section{References}

1. Quintanilla, M.; Liz-Marzan, L.M. Guiding Rules for Selecting a Nanothermometer. Nano Today 2018, 19, 126-145.

2. McCreery, R.L. Raman Spectroscopy for Chemical Analysis; 2000.

3. Yin, Z.F.; Wu, L.; Yang, H.G.; Su, Y.H. Recent progress in biomedical applications of titanium dioxide. Phys. Chem. Chem. Phys. 2013, 15, 4844-4858.

4. Neumann, P.; Dolde, I.J.F.; Burk, C.; Reuter, R.; Waldherr, G.; Honert, J.; Wolf, T.; Brunner, A.; Shim, J.H.; Suter, D.; et al. Thermometry at the Nanoscale; 2019.

5. McCabe, K.M.; Hernandez, M. Molecular Thermometry. Pediatric Res. 2010, 67, 469-475.

6. Bai, T.; Gu, N. Micro/Nanoscale Thermometry for Cellular Thermal Sensing. Small 2016, 12, 4590-4610.

7. Uchiyama, S.; Gota, C.; Tsuji, T.; Inada, N. Intracellular temperature measurements with fluorescent polymeric thermometers. Chem. Commun. 2017, 53, 10976-10992.

8. Griffiths, J.E.; Malyj, M. Stokes/Anti-Stokes Raman Vibrational Temperatures: Reference Materials, Standard Lamps, and Spectrophotometric Calibrations. Appl. Spectrosc. 1983, 37, 315-333.

9. Beechem, T.E.; Serrano, J.R. Raman Thermometry of Microdevices: Choosing a Method to Minimize Error. Spectroscopy 2011, 26, 36-44.

10. Tuschel, D. Raman Thermometry: Understanding the Mathematics to Better Design Raman Measurements. Spectroscopy 2019, 34, 8-13.

11. Serrano, J.R.; Phinney, L.M.; Kearney, S.P. Micro-Raman evaluation of polycrystalline silicon MEMS devices. MRS Online Proc. Libr. Arch. 2005, 890. doi:10.1557/PROC-0890-Y08-37.

12. Beechem, T.; Christensen, A.; Graham, S.; Green, D. Micro-Raman thermometry in the presence of complex stresses in GaN devices. J. Appl. Phys. 2008, 103, 8.

13. Abel, M.R.; Graham, S.; Serrano, J.R.; Kearney, S.P.; Phinney, L.M. Raman thermometry of polysilicon microelectromechanical systems in the presence of an evolving stress. J. Heat Transf. Trans. ASME 2007, 129, 329-334.

14. Sarua, A.; Bullen, A.; Haynes, M.; Kuball, M. High-resolution Raman temperature measurements in GaAs p-HEMT multifinger devices. IEEE Trans. Electron Devices 2007, 54, 1838-1842.

15. Ahmad, I.; Kasisomayajula, V.; Holtz, M.; Berg, J.M.; Kurtz, S.R.; Tigges, C.P.; Allerman, A.A.; Baca, A.G. Self-heating study of an AlGaN/GaN-based heterostructure field-effect transistor using ultraviolet microRaman scattering. Appl. Phys. Lett. 2005, 86, 3. 
16. Calizo, I.; Balandin, A.A.; Bao, W.; Miao, F.; Lau, C.N. Temperature dependence of the Raman spectra of graphene and graphene multilayers. Nano Lett. 2007, 7, 2645-2649.

17. Rassat, S.D.; Davis, E.J. Temperature-measurement of single levitated microparticles using Stokes antiStokes Raman intensity ratios. Appl. Spectrosc. 1994, 48, 1498-1505.

18. Lundt, N.; Kelly, S.T.; Rodel, T.; Remez, B.; Schwartzberg, A.M.; Ceballos, A.; Baldasseroni, C.; Anastasi, P.A.F.; Cox, M.; Hellman, F.; et al. High spatial resolution Raman thermometry analysis of $\mathrm{TiO}_{2}$ microparticles. Rev. Sci. Instrum. 2013, 84, 7.

19. Gallardo, J.J.; Navas, J.; Zorrilla, D.; Alcantara, R.; Valor, D.; Fernandez-Lorenzo, C.; Martin-Calleja, J. Micro-Raman Spectroscopy for the Determination of Local Temperature Increases in TiO2 Thin Films due to the Effect of Radiation. Appl. Spectrosc. 2016, 70, 1128-1136.

20. Peral, J.; Domenech, X.; Ollis, D.F. Heterogeneous photocatalysis for purification, decontamination and deodorization of air. J. Chem. Technol. Biotechnol. 1997, 70, 117-140.

21. Giarola, M.; Sanson, A.; Monti, F.; Mariotto, G.; Bettinelli, M.; Speghini, A.; Salviulo, G. Vibrational dynamics of anatase TiO2: Polarized Raman spectroscopy and ab initio calculations. Phys. Rev. B 2010, 81, 7.

22. Pascual, J.; Camassel, J.; Mathieu, H. Resolved Quadrupolar Transition in $\mathrm{TiO}_{2}$. Phys. Rev. Lett. 1977, 39, 1490-1493.

23. Fujishima, A.; Honda, K. Electrochemical Photolysis of Water at a Semiconductor Electrode. Nature 1972, 238, 37-38.

24. Michael, G. The Artificial Leaf, Molecular Photovoltaics Achieve Efficient Generation of Electricity from Sunlight. Comments Inorg. Chem. 2006, 12, 93-111.

25. Ohsaka, T.; Izumi, F.; Fujiki, Y. Raman spectrum of anatase, TiOz. J. Raman Spectrosc. 1978, 7, 321-324.

Publisher's Note: MDPI stays neutral with regard to jurisdictional claims in published maps and institutional affiliations.

(C) 2020 by the authors. Submitted for possible open access publication under the terms and conditions of the Creative Commons Attribution (CC BY) license (http://creativecommons.org/licenses/by/4.0/). 